\title{
Phase Evolution of Superconducting Sn-In-Bi Solder Alloys
}

\author{
Tayebeh Mousavi, Canan Aksoy, Chris Grovenor, Susannah Speller
}

\begin{abstract}
Development of $\mathrm{Pb}$-free superconducting solders is of great importance for the superconducting magnet manufacturers who often use $\mathbf{P b}$-Bi alloys as superconducting solders. Here, we study the phase evolution, microstructure and superconducting properties of a number of alloys in the $\mathrm{Pb}$-free $\mathrm{Sn}$-In-Bi system using analytical scanning electron microscopy (SEM), differential thermal analysis (DTA), X-ray Diffraction (XRD) and SQUID magnetometry. All the alloys exhibit melting points below $200^{\circ} \mathrm{C}$ and superconducting transition temperatures above $4.2 \mathrm{~K}$. The choice of initial composition and cooling rate control the microstructure (chemistry, volume fraction, morphology and scale) of these alloys and strongly influence the final superconducting properties. The In-rich $\beta$-phase shows better superconducting properties compared to the other phases in the Sn-In-Bi system. As a result, by increasing indium content in the alloy, volume fraction of the In-rich $\beta$-phase increases leading to lower melting point, higher wettability and better superconducting properties.
\end{abstract}

Index Terms-Superconducting Properties, Phase evolution, Microstructure, Solders.

\section{INTRODUCTION}

$S^{2}$ oldering is one of the widely used techniques for making persistent current joints between technological superconductors such as NbTi wires [1-4]. In this technique, lead-free solders are of great importance as new restrictions on the use of $\mathrm{Pb}$ alloys $[5,6]$ will soon become a serious concern for superconducting magnet manufacturers, as $\mathrm{Pb}-\mathrm{Bi}$ alloys are typically used as superconducting solders. Generally, a leadfree solder candidate must fulfill several requirements such as low melting temperature, satisfactory wettability and suitable thermal and mechanical properties. In addition to the physical and mechanical properties, high superconducting critical current density at the practical magnetic field $(1 \mathrm{~T})$ is of fundamental importance.

The microstructure of the solder materials strongly influences the overall performance of the joints, both in terms of superconducting properties and physical/mechanical properties [7]. Similar to many superconducting materials, in soldering materials, superconducting properties such as $\mathrm{T}_{\mathrm{C}}, \mathrm{J}_{\mathrm{C}}$ and $\mathrm{B}_{\mathrm{C} 2}$ typically vary with composition and the presence of the defects, impurities and secondary phase [4]. Moreover, when a joint is made by the soldering technique, it is important that no holes, cracks or non-superconducting phase is present at the interface of the solder and substrates. As most of the solders are eutectic alloys, they have two-phase microstructures, with one or both phases being a superconductor. In these microstructures, the

T. Mousavi, C. Grovenor, and S. Speller are with the Department of Materials, University of Oxford, Oxford OX1 3PH, United Kingdom (e-mail: tayebeh.mousavi@materials.ox.ac.uk; chris.grovenor@materials.ox.ac.uk; overall superconducting properties of the solder is usually controlled by the properties of the majority phase [8]. As a result, it is necessary that the majority phase of the solder has acceptable superconducting properties to make a successful joint. In some eutectic microstructures, one phase forms a continuous matrix for the other disconnected phases, and they can act as flux pinning sites provided the second phase particles are small enough. However, in these microstructures, the secondary phase is usually larger than the coherence length, and instead the interphase boundaries act as active pinning sites enhancing the critical current density in magnetic field [8].

As the microstructure of the solder plays such a significant role in the overall performance of the joint, it is important to study the phase evolution of the solders during solidification. According to the literature, alloys of Sn and In show higher values of transition temperature $\left(\mathrm{T}_{\mathrm{C}}\right)$ and critical field $\left(\mathrm{B}_{\mathrm{C} 2}\right)$ compared to the other lead-free soldering materials [7]. In our previous work, we found that in In-Sn-Bi system, superconducting properties strongly depend on the present phases in the microstructure [9]. Here, we focus on the phase evolution of various compositions in the lead-free Sn-In-Bi system.

\section{EXPERIMENTAL METHODS}

The following compositions were chosen in different regions of the ternary Sn-In-Bi phase diagram to study:

- $\quad$ Sn-50wt.\%In-30wt.\%Bi (eutectic composition)

- $\quad$ Sn-50wt.\%In-15wt.\%Bi (In-rich)

- $\quad$ Sn-30wt.\%In-20wt.\%Bi (Sn-rich)

These alloys were fabricated from pure $\mathrm{Sn}$, In and $\mathrm{Bi}$ powders (with high purity above $99.9 \%$ ) by weighing the powder and melting the mixture at temperatures up to $300^{\circ} \mathrm{C}$ on a hot-plate. The melt was sucked into pre-heated $2 \mathrm{~mm}$ diameter cylindrical quartz tubes and cooled in air.

The morphology and elemental/phase maps of the samples were characterized using a scanning electron microscope (SEM) and energy-dispersive $\mathrm{x}$-ray (EDX) respectively in a Jeol 5510 SEM with OI SDD detector operating at $20 \mathrm{kV}$ accelerating voltage. The phase maps were generated by the OI Aztec software, and the average composition of each phase and the phase fraction were calculated from the reconstructed spectra from each phase in the map. X-ray Diffraction (XRD) analysis was carried out using $\mathrm{Cu}$ Ka radiation in a Philips $\mathrm{h}-2 \mathrm{~h}$ diffractometer and a Philips X'Pert MRD four-circle diffractometer.

The melting temperature and phase evolution of the solders during solidification were analyzed by differential scanning

susannah.speller@materials.ox.ac.uk, and Canan Aksoy is with Electronic and Communication Department, Faculty of Technology, Karadeniz Technical University, Terbzon, Turkey, aksoycanan0@gmail.com. 
calorimeter (DSC) using a Perkin Elmer Diamond DSC at a heating/cooling rate of $10 \mathrm{~K} / \mathrm{min}$ under nitrogen atmosphere. For this measurement, about $11 \mathrm{mg}$ of the sample was sealed in a hermetic aluminum pan. The superconducting properties were investigated by measuring magnetization using a SQUID system. M-T curves were taken in a small measurement field of $0.01 \mathrm{~T}$ to determine the $\mathrm{T}_{\mathrm{C}} . \mathrm{J}_{\mathrm{C}}$ values were extracted from full hysteresis curves taken at a range of selected temperatures. For these measurements, all the samples were prepared with the same size and shape (cylinders with $2 \mathrm{~mm}$ diameter and $2.5 \mathrm{~mm}$ in length) and were carefully aligned with their axis parallel to the applied magnetic field. To extract $\mathrm{J}_{\mathrm{C}}$ from magnetic hysteresis loops, Bean's model and self-field corrections were applied [10].

\section{RESULTS}

\section{A. Sample Sn-50wt.\%In-30wt.\%Bi}

Fig 1 shows the elemental maps of the sample Sn-50wt.\%In$35 \mathrm{wt} . \% \mathrm{Bi}$ along with the phase map and DTA data. This sample has a nominal composition of the ternary eutectic point in the Sn-In-Bi system. The EDX elemental maps show that there is a clear elemental segregation for $\mathrm{Sn}, \mathrm{Bi}$ and In.

The phase map extracted from the EDX mapping data shows that the microstructure consists of three distinct phases. From the reconstructed EDX spectra, the composition of each phase was obtained and compared to the ternary $\mathrm{Sn}-\mathrm{In}-\mathrm{Bi}$ phase diagram. It was found that the microstructure contains $\mathrm{BiIn}_{2}$ as the majority phase in the matrix, with a network of $\gamma(\mathrm{Sn}$-rich) and $\beta$ (In-rich) phases formed next to each other within the $\mathrm{BiIn}_{2}$ matrix on a scale of about $20 \mu \mathrm{m}$. The presence of these three phases has been also confirmed by XRD as shown in Fig.1g.

DTA data confirms only one sharp transition at $55^{\circ} \mathrm{C}$ upon heating and cooling indicating that the alloy has solidified by the following ternary eutectic reaction.

$$
\mathrm{L} \rightarrow \gamma+\beta+\mathrm{BiIn}_{2}\left(\text { at } 55^{\circ} \mathrm{C}\right)
$$

It can be concluded that in this sample, liquid having the composition of Sn-50wt.\%In-35wt.\%Bi directly solidifies into solid phases $\gamma$ (Sn-30In-20Bi), $\beta$ (Sn-60In-20Bi) and BiIn 2 .

Similar microstructures have previously been reported for the ternary eutectic composition in this system [11,12]. Ruggerio et al found a microstructure consisting of regions of regular binary structure of Sn-rich and In-rich phases within a matrix with a composition close to BiIn 2 . They also found that the chemistry and scale of this microstructure strongly depends on the cooling rate, and the phase spacing $(\lambda)$ is correlated to the cooling rate $(\mathrm{R})$ by the well-known relationship for eutectic solidification $\lambda^{2} \mathrm{R}=$ constant.
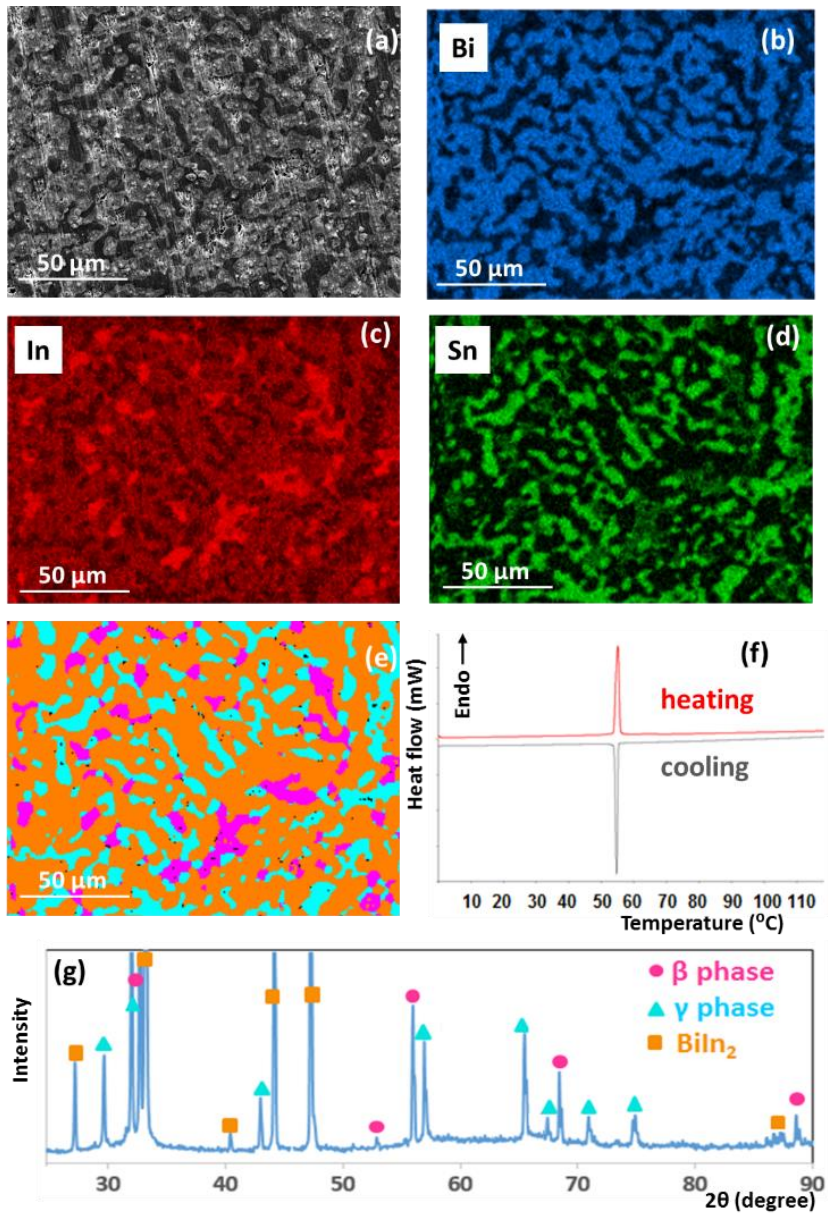

Fig. 1. (a) Electron-backscattered image, (b-d) bismuth, indium and tin maps, (e) Phase map, (f) heating and cooling DSC curves and (g) XRD pattern of sample Sn-50wt \% In-35wt\%Bi. In the phase map, orange represents BiIn ${ }_{2}$, blue represents $\gamma$ phase and pink represents $\beta$ phase.

\section{B. Sample Sn-50wt.\%In-15wt.\%Bi}

This sample was chosen to engineer a microstructure with a majority phase of $\beta$. EDX analysis (Fig. 2) confirms that there are elemental segregation for all three elements of tin, indium and bismuth resulting three phases of $\gamma, \beta$ and $\mathrm{BiIn}_{2}$, with the majority $\beta$ phase forming a continuous matrix.

The DTA curve from this alloy shows that the following three endothermic transformations happen during cooling from the molten state.
i) $\quad \mathrm{L} \rightarrow \beta \quad\left(\right.$ from $80^{\circ} \mathrm{C}$ to $\left.68^{\circ} \mathrm{C}\right)$
ii) $\quad \mathrm{L} \rightarrow \gamma+\beta$ (from $68^{\circ} \mathrm{C}$ to $50^{\circ} \mathrm{C}$ )
iii) $\quad \mathrm{L} \rightarrow \gamma+\beta+\mathrm{BiIn}_{2}\left(\right.$ at $\left.50^{\circ} \mathrm{C}\right)$

During the solidification of the liquid with the composition of Sn-50wt\%In-15wt\%Bi, primary $\beta$ phase nucleates first at $80^{\circ} \mathrm{C}$ and grow dendritically until the temperature falls to about $68^{\circ} \mathrm{C}$. At this temperature, a binary eutectic reaction occurs $(\mathrm{L} \rightarrow \gamma+\beta$ ) leading to the formation of $\gamma$ and $\beta$ phases next to each other. Finally at about $50^{\circ} \mathrm{C}$, the remaining liquid solidifies by the ternary eutectic reaction ( $\mathrm{L} \rightarrow \gamma+\beta+\mathrm{BiIn}_{2}$ ), resulting in a three phase microstructure. In this microstructure, $\mathrm{BiIn}_{2}$ is the last phase to be formed and $\beta$ phase is formed at all stages. According to EDX data, the composition of the $\beta$ phase, which is the majority phase in the matrix, is In- $15 \mathrm{wt} . \% \mathrm{Bi}-20 \mathrm{wt} . \% \mathrm{Sn}$. 

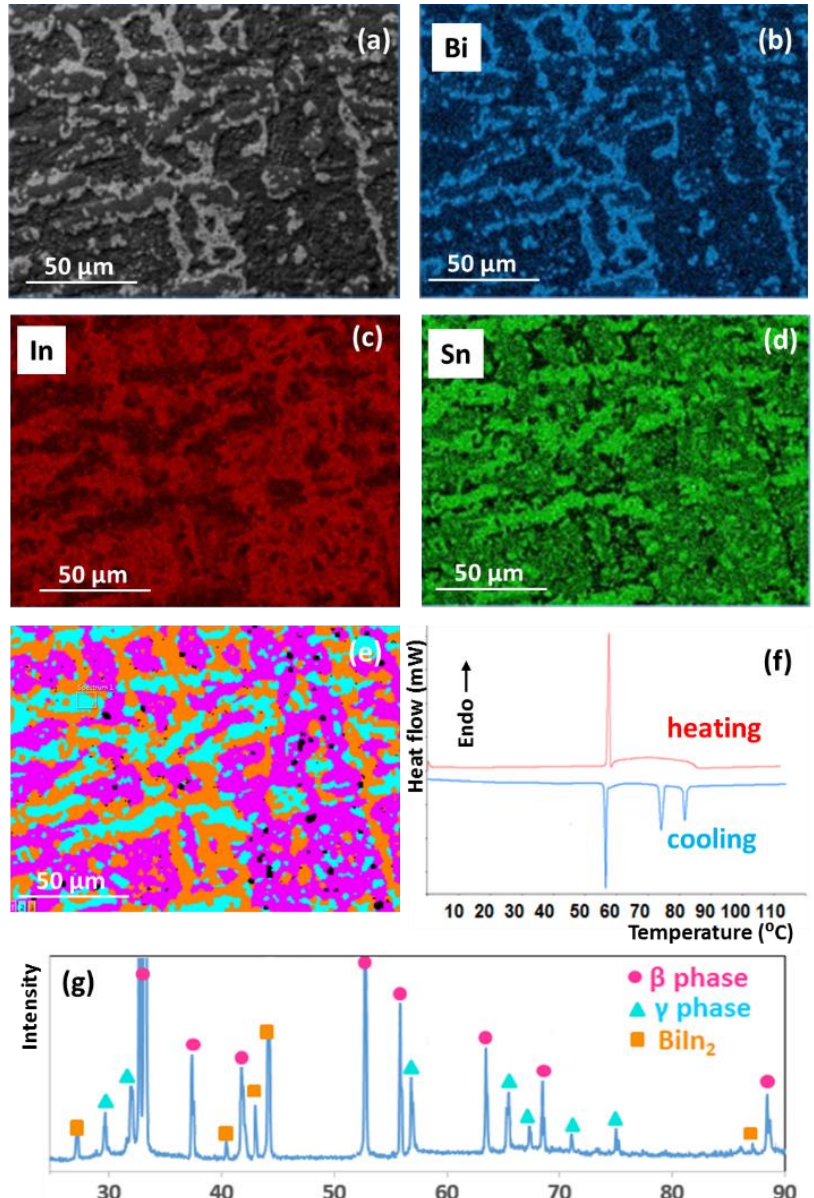

$10203040 \quad 5060708090100110$
Temperature $\left({ }^{\circ} \mathrm{C}\right)$

30

40

50

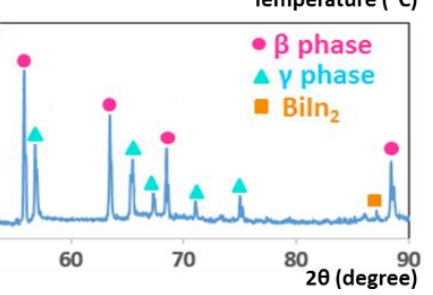

Fig. 2. (a) Electron-backscattered image, (b-d) bismuth, indium and tin maps, (e) Phase map, (f) heating and cooling DSC curves and (g) XRD pattern of sample $\mathrm{Sn}-50 \mathrm{wt} \% \mathrm{In}-15 \mathrm{wt} \% \mathrm{Bi}$. In the phase map, orange represents $\mathrm{BiIn}_{2}$, blue represents $\gamma$ phase and pink represents $\beta$ phase.

\section{Sample Sn-30wt.\%In-20wt.\%Bi}

Sample Sn-30wt.\%In-20wt.\%Bi contains an overall composition richer in $\mathrm{Sn}$ and was chosen to form a microstructure with the majority phase of $\gamma$. The EDX elemental mapping analysis (Fig. 3b-d) shows that indium is dissolved uniformly over the solder, and only bismuth and tin are segregated. The sample has a two-phase microstructure consisting of BiIn precipitates distributed on a relatively fine scale within a matrix of the $\gamma$-phase.

DTA analysis of this sample exhibits two endothermic transitions over cooling which correspond to the following phase transitions;

i) $\quad \mathrm{L} \rightarrow \gamma \quad\left(\right.$ from $128^{\circ} \mathrm{C}$ to $68^{\circ} \mathrm{C}$

ii) $\quad \mathrm{L} \rightarrow \gamma+\mathrm{BiIn}$ (from $68^{\circ} \mathrm{C}$ )

The first transition is a large but narrow peak at $128^{\circ} \mathrm{C}$ and to the beginning of the solidification of primary $\gamma$ phase. The other smaller peak in the cooling data at lower temperature corresponds to the start of the binary eutectic phase transition leading to the formation of $\gamma+$ BiIn phases.
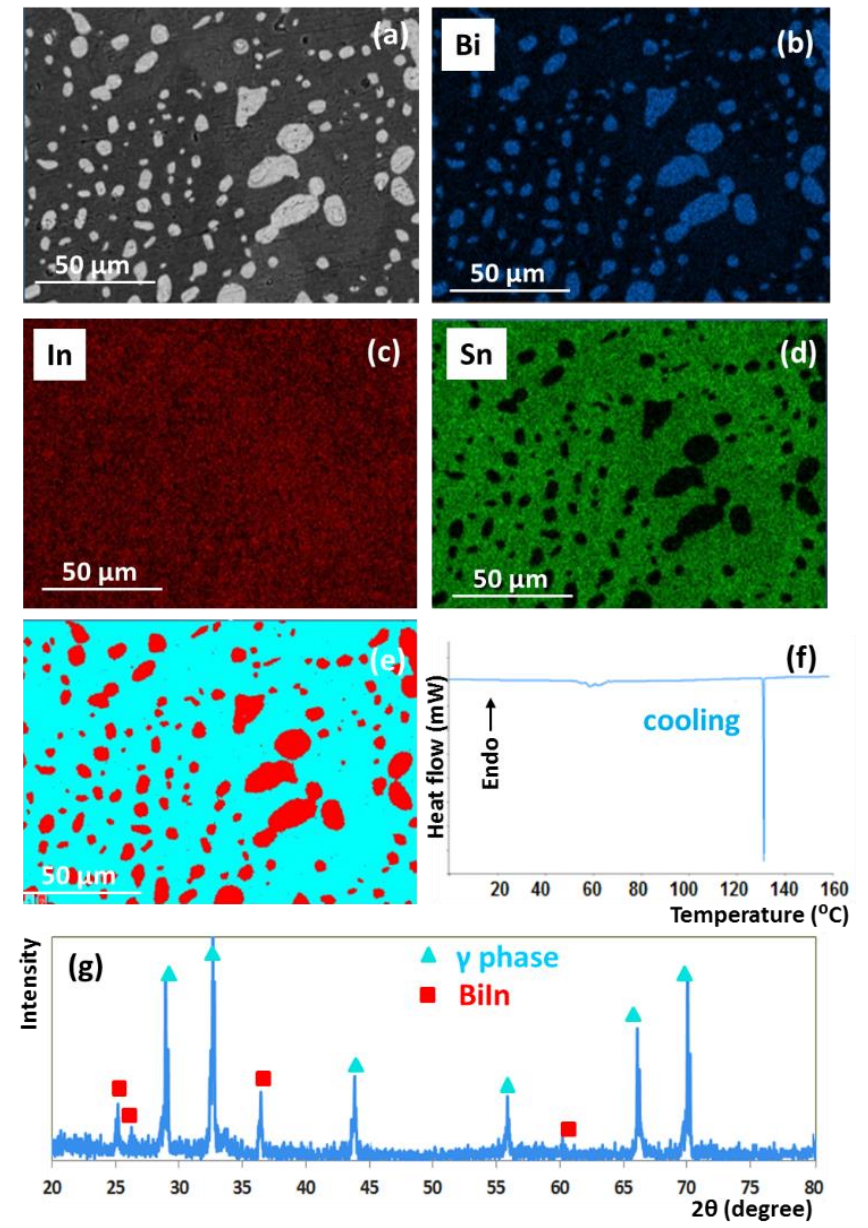

Fig. 3. (a) Electron-backscattered image, (b-d) bismuth, indium and tin maps, (e) Phase map, (f) cooling DSC curves and (g) XRD pattern of sample Sn$30 \mathrm{wt} \%$ In-20wt\%Bi. In the phase map, red represents BiIn, blue represents $\gamma$ phase.

\section{Superconducting properties}

Fig. 4 along with Table 1 present superconducting parameters $\left(\mathrm{T}_{\mathrm{C}}, \mathrm{J}_{\mathrm{C}}\right.$ and hysteresis loops) and microstructural information of the three studied samples. Sample Sn-30In-20Bi with the majority phase of $\gamma$ shows the lowest transition temperature, whereas both the other two samples show $T_{C}$ values above $4.2 \mathrm{~K}$. Sample Sn-50In-15Bi, which contains $\beta$ phase as the majority phase in the matrix, exhibits the highest $\mathrm{T}_{\mathrm{C}}$. This is consistent with the literature where the binary Sn-In $\beta$-phase has higher $T_{C}$ values $(6.1 \mathrm{~K})$ compared to the binary Sn-In $\gamma$-phase $\left(\mathrm{T}_{\mathrm{C}}=4.5 \mathrm{~K}\right)$ and $\mathrm{BiIn}_{2}\left(\mathrm{~T}_{\mathrm{C}}=5.5 \mathrm{~K}\right)$ [13]. In agreement with [9], these results show that in a multi-phase microstructure, the majority phase controls overall superconducting parameters of the sample. In Fig $4 \mathrm{~b}$, critical current density of these alloys are shown with the highest value for sample Sn-50In-15Bi consisting of a matrix of $\beta$. In spite of the fact that eutectic sample has higher $\mathrm{T}_{\mathrm{C}}$ value and similar $\mathrm{B}_{\mathrm{C} 2}$ value compared to those of Sn-rich sample ( $\mathrm{Sn}$-30In-20Bi), it shows much lower critical current density. This can be due to extrinsic parameters influencing critical current density such as microstructural features. As found by the SEM phase map analysis, the Sn-rich sample with the majority phase of $\gamma$, has 
very small precipitates of a secondary phase which might lead to an increase in the active pinning sites.
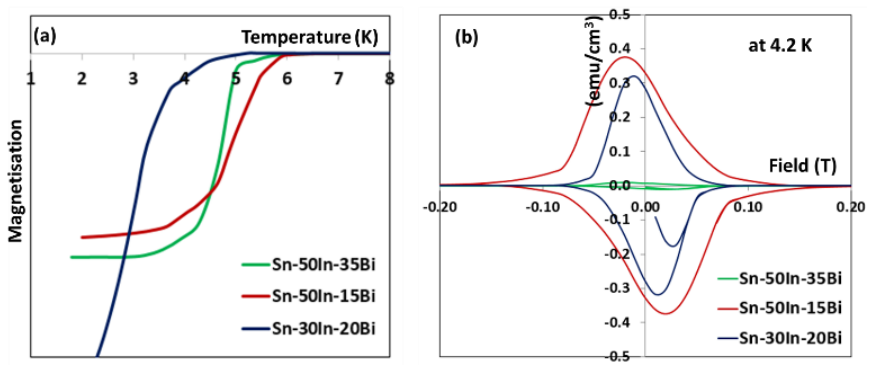

Fig. 4. Superconducting properties of three Sn-In-Bi alloys studied in this work. (a) Magnetization as a function of temperature in a small measurement field of $0.01 \mathrm{~T}$, (b) Hysteresis loops of the samples.

Table 1-A summary of the properties of the studied Sn-In-Bi alloys

\begin{tabular}{|c|c|c|c|c|c|c|c|}
\hline \multirow{2}{*}{ Samples } & \multirow{2}{*}{$\begin{array}{c}\mathbf{T}_{\mathbf{C}}(\mathbf{K}) \\
\text { at } \\
0.01 \mathrm{~T}\end{array}$} & \multirow{2}{*}{$\begin{array}{l}\mathrm{B}_{\mathrm{C} 2}(\mathrm{~T}) \\
\text { at } 4.2 \mathrm{~K}\end{array}$} & \multirow{2}{*}{$\begin{array}{c}J_{C} \\
(\mathrm{~A} / \mathrm{m} 2) \\
\text { at } 4.2 \mathrm{~K}- \\
0.01 \mathrm{~T} \\
\end{array}$} & \multicolumn{4}{|c|}{ Phase (Vol \%) } \\
\hline & & & & $\beta$ & $\gamma$ & BiIn $_{2}$ & BiIn \\
\hline Sn-50In-35Bi & 4.9 & 0.09 & $3 \times 10^{4}$ & 23 & 21 & 56 & - \\
\hline Sn-50In-15Bi & 5.5 & 0.18 & $12.8 \times 10^{7}$ & 66 & 16 & 18 & - \\
\hline Sn-30In-20Bi & 3.8 & 0.075 & $10.5 \times 10^{7}$ & - & 75 & - & 25 \\
\hline
\end{tabular}

\section{CONCLUSION}

Three alloys (Sn-30wt.\%In-20wt.\%Bi, Sn-50wt.\%In$15 \mathrm{wt} . \% \mathrm{Bi}$ and $\mathrm{Sn}-50 \mathrm{wt} . \%$ In-30wt.\%Bi) were studied to evaluate their potential as a replacement for the toxic $\mathrm{Pb}$ containing solders. These three compositions were chosen to engineer different microstructures, especially different phases $\left(\gamma, \beta\right.$ and $\left.\mathrm{BiIn}_{2}\right)$ as the majority in the matrix to understand how phase evolution and microstructure influence superconducting properties.

It was found that all the alloys have melting points below $200^{\circ} \mathrm{C}$ and superconducting transition temperatures above $4 \mathrm{~K}$. By increasing In content in the alloy (ie the alloys in the In-rich corner of the phase diagram), lower melting point, higher wettability and better superconducting properties can be achieved. Although, as eutectic composition, sample Sn-50In$35 \mathrm{Bi}$ shows the lowest melting point of $55^{\circ} \mathrm{C}$ in the ternary $\mathrm{Sn}$ In-Bi system with the molten alloy solidifying directly by the ternary eutectic reaction to $\beta, \gamma$ and $\mathrm{BiIn}_{2}$, the critical density is not as high as other two alloys in this system. The intrinsic superconducting properties such as $\mathrm{T}_{\mathrm{C}}$ are controlled by the properties of the majority phase. As a result, sample Sn-50In$15 \mathrm{Bi}$ with the majority phase of $\beta$ shows the highest superconducting parameters. However the $\mathrm{J}_{\mathrm{C}}$ value is still significantly lower than the PbBi alloys, and an improvement in these parameters is necessary for a reliable replacement for the $\mathrm{Pb}$-containing solders.

\section{REFERENCES}

[1] C.A. Swenson, W.D. Markiewicz, Persistent joint development for high field NMR, IEEE Trans. Appl. Supercond. Vol. 9, pp. 185-8, 1999.
[2] R.F Hornton, Superconducting joint for superconducting wires and coils (U.S. Patent 5410288), 1986.

[3] T. Fukuzaki, H. Maeda, S. Matsumoto, S. Nimori, S. Yokoyama, T. Kiyoshi, Development of a Superconducting Joint for High Field NMR, IEEE Trans. Appl. Supercond. Vol. 16, pp. 1547-9, 2006.

[4] J. Cheng, J. Liu, Z. Ni, C. Cui, S. Chen, S. Song, L. Li, Y. Dai, Q. Wang, Fabrication of NbTi Superconducting Joints for $400-\mathrm{MHz}$ NMR Application, IEEE Trans. Appl. Supercond. Vol. 22, pp. 4300205, 2012.

[5] Official Journal of the European Union, Directive 2011/65/EU, 2011.

[6] Official Journal of the European Union, Directive 2014/9/EU, 2014.

[7] G. Brittles, T. Mousavi, C. Aksoy, C. Grovenor, S. Speller, Persistent current joints between technological superconductors, Superconducting Science and Technology, Vol.12, pp. 1243, 2015.

[8] J.D. Livingston, Superconducting Properties of Eutectics, J. Appl. Phys. Vol. 38, pp. 2408, 1967.

[9] T. Mousavi, C. Aksoy, C. Grovenor, S. Speller, Microstructure and superconducting properties of Sn-In and Sn-In-Bi alloys as Pb-free superconducting solders, Superconducting Science and Technology, Vol. 29, pp. 015012, 2016.

[10] C.P. Bean, Magnetization of high-field superconductors, Rev. Mod. Phys. Vol. 36 pp. 31-39, 1964.

[11] H. Kabassis, J.W. Rutter, W.C. Winegard, Metallurgical Transactions A, Vol. 15, pp. 1515-1517, 1984.

[12] H. Kabassis, J. Rutter, W. Winegard, Materials Science and Technology, Vol. 2, pp. 985, 1986.

[13] L. I. Berger and B. W. Roberts, Properties of superconductors, CRC Press, Boca Raton, 1997. 